UNIVERSITY OF GOTHENBURG

SCHOOL OF BUSINESS, ECONOMICS AND LAW

WORKING PAPERS IN ECONOMICS

No 545

\begin{abstract}
A Markov Copula Model of Portfolio Credit Risk with Stochastic Intensities and Random Recoveries
\end{abstract}

Tomasz R. Bielecki, Areski Cousin, Stéphane Crépey and Alexander Herbertsson

October 2012

ISSN 1403-2473 (print)

ISSN 1403-2465 (online) 


\title{
A Markov Copula Model of Portfolio Credit Risk with Stochastic Intensities and Random Recoveries
}

\author{
Tomasz R. Bielecki ${ }^{1, *}$, Areski Cousin ${ }^{2 \dagger}$, \\ Stéphane Crépey ${ }^{3, \ddagger}$, Alexander Herbertsson ${ }^{4, \S}$ \\ ${ }^{1}$ Department of Applied Mathematics \\ Illinois Institute of Technology \\ Chicago, IL 60616, USA \\ ${ }^{2}$ Université de Lyon, \\ Université Lyon 1, Laboratoire SAF, EA 2429, \\ Institut de Science Financière et d'Assurances, \\ 50 Avenue Tony Garnier, 69007 Lyon, France. \\ ${ }^{3}$ Laboratoire Analyse et Probabilités \\ Université d'Évry Val d'Essonne \\ 91037 Évry Cedex, France \\ ${ }^{4}$ Centre for finance/Department of Economics \\ University of Gothenburg \\ SE 40530 Göteborg, Sweden
}

October 4, 2012

\begin{abstract}
In [4], the authors introduced a Markov copula model of portfolio credit risk. This model solves the top-down versus bottom-up puzzle in achieving efficient joint calibration to single-name CDS and to multi-name CDO tranches data. In [4, we studied a general model, that allows for stochastic default intensities and for random recoveries, and we conducted empirical study of our model using both deterministic and stochastic default intensities, as well as deterministic and random recoveries only. Since, in case of some "badly behaved" data sets a satisfactory calibration accuracy can only be achieved through the use of random recoveries, and, since for important applications, such as CVA computations for credit derivatives, the use of stochastic intensities is advocated by practitioners, efficient implementation of our model that would account for these
\end{abstract}

\footnotetext{
${ }^{*}$ The research of T.R. Bielecki was supported by NSF Grant DMS-0604789 and NSF Grant DMS-0908099.

${ }^{\dagger}$ The research of A. Cousin benefited from the support of the DGE and the ANR project Ast\&Risk.

${ }^{\ddagger}$ The research of S. Crépey benefited from the support of the 'Chaire Risque de crédit', Fédération Bancaire Française.

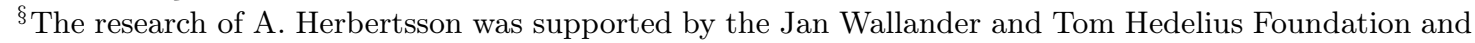
by Vinnova.
} 
two issues is very important. However, the details behind the implementation of the loss distribution in the case with random recoveries were not provided in [4. Neither were the details on the stochastic default intensities given there. This paper is thus a complement to [4, with a focus on a detailed description of the methodology that we used so to implement these two model features: random recoveries and stochastic intensities.

Keywords: Portfolio Credit Risk, Markov Copula Model, Common Shocks, Stochastic Spreads, Random Recoveries.

\section{Introduction}

In [4] we introduced a common-shock Markov copula model of default times providing an effective joint calibration to single-name CDS and multi-name CDO tranches data. In this sense this model solves the portfolio credit risk top-down bottom-up puzzle [6]. For earlier, partial progress in this direction, see [11, 12, 13, 17] and the discussion in [4]. The main model feature is the use of common jumps to default, triggered by common shocks, as a powerful dependence device also compatible with the Markov copula properties [9], the latter being required to decouple the calibration of the individual (single-name) model parameters from the few model dependence parameters.

On the other hand, the model presented in [4] is also a fully dynamic model in which the critical issue of modeling counterparty risk embedded in credit derivatives, and consequently the issue of computation and hedging of CVA, can be consistently and practically addressed [2, 5, 7, 10]. However, as it was emphasized in a June 2011 Bank of International Settlements press release that "During the financial crisis of 2007-09, roughly two-thirds of losses attributed to counterparty credit risk were due to CVA losses and only about one-third were due to actual defaults". In other words, the volatility of CVA matters as much as its level. Consequently (and also to be consistent with the optional nature of the CVA), for CVA computations for credit derivatives, practitioners strongly advocate the use of stochastic default intensities. Moreover, in case of some "badly behaved" data sets, a satisfying calibration accuracy can only be achieved by resorting to random recoveries.

In order to respond to these considerations, in the present paper, which is a companion paper to [4, we provide more background, implementation hints, as well as numerical illustration accounting for these two features which are important for applications: stochastic spreads and random recoveries. Section 2 reviews the model of default times which is used, including the specification of the stochastic intensities and recoveries. Regarding the default intensities, we resort to time-inhomogenous affine processes with time-dependent piecewise-constant mean-reversion level, resulting in analytical tractability (see below) and calibration flexibility (of the term-structure of CDS spreads in particular). For tractability reasons, random recoveries are taken to be independent between them, as well as independent from everything else in the model.

Section 3 is about pricing in this setup. In particular pricing of CDS, as well as of CDO tranches, ultimately boils down here to computations of Laplace transform for time-inhomogenous affine processes. Proposition 3.2 shows how this can be done explicitly, exploiting the piecewiseconstant mean-reversion structure of the intensities.

Again, effective joint calibration of this model to CDS and CDO data is an important achievement. Section 4 reviews in detail and illustrates the calibration methodology, regarding in particular the stochastic affine intensities and random recovery specifications which are used.

In the rest of the paper we consider a risk neutral pricing model $(\Omega, \mathcal{F}, \mathbb{P})$, for a filtration $\mathcal{F}=\left(\mathcal{F}_{t}\right)_{t \in[0, T]}$ which will be specified below, and where $T \geq 0$ is a fixed time horizon. We denote $\mathbb{N}_{n}=\{1, \ldots, n\}$ and we let $\mathcal{N}_{n}$ denote the set of all subsets of $\mathbb{N}_{n}$ where $n$ represents the number of obligors in the underlying credit portfolio. We also let $\tau_{i}$ and $H_{t}^{i}=\mathbb{1}_{\left\{\tau_{i} \leq t\right\}}$ denote the default time of name $i=1,2, \ldots, n$ and the corresponding indicator process. 


\section{Model of Default Times}

We recall a common shocks portfolio credit risk model of 4 . In order to describe the defaults we define a certain number $m$ (typically small: a few units) of groups $I_{j} \subseteq \mathcal{N}_{n}$, of obligors who are likely to default simultaneously, for $j \in \mathbb{N}_{m}$. More precisely, the idea is that at every time $t$, there will be a positive probability that the survivors of the group of obligors $I_{j}$ (obligors of group $I_{j}$ still alive at time $t$ ) default simultaneously. Let $\mathcal{I}=\left\{I_{1}, \ldots, I_{m}\right\}, \mathcal{Y}=\left\{\{1\}, \ldots,\{n\}, I_{1}, \ldots, I_{m}\right\}$. Given non-negative constants $a, c$ and non-negative deterministic functions $b_{Y}(t)$ for $Y \in \mathcal{Y}$, let a group intensity process $X^{Y}$ be defined in the form of an extended CIR process as: $X_{0}^{Y}$ a given constant, and for $t \in[0, T]$

$$
d X_{t}^{Y}=a\left(b_{Y}(t)-X_{t}^{Y}\right) d t+c \sqrt{X_{t}^{Y}} d W_{t}^{Y}
$$

where the Brownian motions $W^{Y}$ are independent. We refer the reader to [7] for a two-obligors preliminary version of this model dedicated to valuation and hedging of counterparty risk on a CDS. Consistently with the discussion of Section 1, the numerical results of [7] illustrate that such affine specifications of the intensities are appropriate for dealing with counterparty credit risk.

Remark 2.1 The (simpler) case of deterministic time-dependent intensities will also be used in the numerical experiments. It can essentially be thought of as letting $c$ vanish in (1D), so we will not make any specific theoretical developments in this regard, referring the reader if need be to [4] about this.

For $\mathbf{k}=\left(k_{1}, \ldots, k_{n}\right) \in\{0,1\}^{n}$, we introduce $\operatorname{supp}(\mathbf{k})=\left\{i \in \mathbb{N}_{n} ; k_{i}=1\right\}$ and $\operatorname{supp}^{c}(\mathbf{k})=$ $\left\{i \in \mathbb{N}_{n} ; k_{i}=0\right\}$. Hence, $\operatorname{supp}(\mathbf{k})$ denotes the obligors who have defaulted in the portfolio-state $\mathbf{k}$ and similarly $\operatorname{supp}^{c}(\mathbf{k})$ are the survived names in state $\mathbf{k}$. Given $\mathbf{X}=\left(X^{Y}\right)_{Y \in \mathcal{Y}}$, we aim for a model in which the predictable intensity of a jump of $\mathbf{H}=\left(H^{i}\right)_{i \in \mathbb{N}_{n}}$ from $\mathbf{H}_{t-}=\mathbf{k}$ to $\mathbf{H}_{t}=\mathbf{l}$, with $\operatorname{supp}(\mathbf{k}) \varsubsetneqq \operatorname{supp}(\mathbf{l})$ in $\{0,1\}^{n}$, would be given by

$$
\sum_{\left\{Y \in \mathcal{Y} ; \mathbf{k}^{Y}=\mathbf{l}\right\}} X_{t}^{Y}
$$

where $\mathbf{k}^{Y}$ denotes the vector obtained from $\mathbf{k}=\left(k_{i}\right)_{i \in \mathbb{N}_{n}}$ by replacing the components $k_{i}, i \in Y$, by numbers one. The intensity of a jump of $\mathbf{H}$ from $\mathbf{k}$ to $\mathbf{l}$ at time $t$ is thus equal to the sum of the intensities of the groups $Y \in \mathcal{Y}$ such that, if the default of the survivors in group $Y$ occurred at time $t$, the state of $\mathbf{H}$ would move from $\mathbf{k}$ to $\mathbf{l}$.

This is achieved by constructing $\mathbf{H}$ through an $\mathbf{X}$-related change of probability measure, starting from a continuous-time Markov chain with intensity one. As a result (see [4]), the pair-process $(\mathbf{X}, \mathbf{H})$ is a Markov process with respect to the filtration $\mathcal{F}$ generated by the Brownian Motion $\mathbf{W}$ and the random measure counting the jumps of $\mathbf{H}$, with infinitesimal generator $\mathcal{A}$ of $(\mathbf{X}, \mathbf{H})$ acting on every function $u=u(t, \mathbf{x}, \mathbf{k})$ with $t \in \mathbb{R}_{+}, \mathbf{x}=\left(x_{Y}\right)_{Y \in \mathcal{Y}}$ and $\mathbf{k}=\left(k_{i}\right)_{i \in \mathbb{N}_{n}}$ as

$$
\mathcal{A}_{t} u(t, \mathbf{x}, \mathbf{k})=\sum_{Y \in \mathcal{Y}}\left(a\left(b_{Y}(t)-x_{Y}\right) \partial_{x_{Y}} u(t, \mathbf{x}, \mathbf{k})+\frac{1}{2} c^{2} x_{Y} \partial_{x_{Y}^{2}}^{2} u(t, \mathbf{x}, \mathbf{k})+x_{Y} \delta u^{Y}(t, \mathbf{x}, \mathbf{k})\right)
$$

where we denote

$$
\delta u^{Y}(t, \mathbf{x}, \mathbf{k})=u\left(t, \mathbf{x}, \mathbf{k}^{Y}\right)-u(t, \mathbf{x}, \mathbf{k})
$$

\subsection{Markov Copula Properties}

Note that the SDEs for processes the $X^{Y}$ have the same coefficients except for $b_{Y}(t)$, to the effect that for $i \in \mathbb{N}_{n}$,

$$
X^{i}:=\sum_{\mathcal{Y} \ni Y \ni i} X^{Y}=X^{\{i\}}+\sum_{\mathcal{I} \ni I \ni i} X^{I}
$$


is again an extended CIR process, with parameters $a, c$ and

$$
b_{i}(t):=\sum_{\mathcal{Y} \ni Y \ni i} b_{Y}(t)=b_{\{i\}}(t)+\sum_{\mathcal{I} \ni I \ni i} b_{I}(t),
$$

driven by an $\mathcal{F}$-Brownian motion $W^{i}$ such that

$$
\sqrt{X_{t}^{i}} d W_{t}^{i}=\sum_{Y \ni i} \sqrt{X_{t}^{Y}} d W_{t}^{Y}, d W_{t}^{i}=\sum_{Y \ni i} \frac{\sqrt{X_{t}^{Y}}}{\sqrt{\sum_{Y \ni i} X_{t}^{Y}}} d W_{t}^{Y} .
$$

The fact that $W^{i}$ defined by (6) is an $\mathcal{F}$-Brownian motion results from Paul Lévy's characterization of a Brownian motion as a continuous local martingale with bracket process equal to time $t$. One can then check, as is done in 4, that the so-called Markov copula property holds (see 9]), in the sense that for every $i \in \mathbb{N}_{n},\left(X^{i}, H^{i}\right)$ is an $\mathcal{F}$ - Markov process admitting the following generator, acting on functions $v_{i}=v_{i}\left(t, x_{i}, k_{i}\right)$ with $\left(x_{i}, k_{i}\right) \in \mathbb{R} \times\{0,1\}$ :

$$
\begin{aligned}
& \mathcal{A}_{t}^{i} v_{i}\left(t, x_{i}, k_{i}\right)= \\
& \quad a\left(b_{i}(t)-x_{i}\right) \partial_{x_{i}} v_{i}\left(t, x_{i}, k_{i}\right)+\frac{1}{2} c^{2} x_{i} \partial_{x_{i}^{2}}^{2} v_{i}\left(t, x_{i}, k_{i}\right)+x_{i}\left(v_{i}\left(t, x_{i}, 1\right)-v_{i}\left(t, x_{i}, k_{i}\right)\right) .
\end{aligned}
$$

Also, the $\mathcal{F}$ - intensity process of $H^{i}$ is given by $\left(1-H_{t}^{i}\right) X_{t}^{i}$. In other words, the process $M^{i}$ defined by,

$$
M_{t}^{i}=H_{t}^{i}-\int_{0}^{t}\left(1-H_{s}^{i}\right) X_{s}^{i} d s,
$$

is an $\mathcal{F}$-martingale. Finally, the conditional survival probability function of name $i \in \mathbb{N}_{n}$ is given by, for every $t_{i}>t$,

$$
\mathbb{P}\left(\tau_{i}>t_{i} \mid \mathcal{F}_{t}\right)=\mathbb{E}\left\{\exp \left(-\int_{t}^{t_{i}} X_{s}^{i} d s\right) \mid X_{t}^{i}\right\}
$$

\section{Pricing}

Regarding the dynamics of CIR intensity processes, we assume in the sequel that the mean-reversion functions $b_{Y}(t)$ are piecewise-constant with respect to a time tenor $\left(T_{k}\right)_{k=1, \ldots, M}$. So, for every $k=1 \ldots M$,

$$
b_{Y}(t)=b_{Y}^{(k)}, t \in\left[T_{k-1}, T_{k}\right) .
$$

where $T_{0}=0$. The time tenor $\left(T_{k}\right)$ is a set of pillars corresponding to standard CDS maturities. In this framework, we are able to provide explicit expressions for survival probabilities of triggering events, which are the main building blocks in the calculation of CDS and CDO tranche spreads.

\subsection{Survival Probabilities of Trigger-Events}

In this subsection, we provide an analytical expression for survival probabilities in an extended CIR intensity model with piecewise constant mean-reversion parameter. This result derives from Prop. 8.2 in [7. Contrary to the latter result which involves recursively-defined parameters, Proposition 3.2 provides closed form expressions for survival probabilities and conditional density functions. This allows us to compute CDS and CDO tranche spreads almost as fast as in a (deterministic) piecewise constant intensity model.

Let $X$ be an extended CIR process with dynamics

$$
d X_{t}=a\left(b(t)-X_{t}\right) d t+c \sqrt{X_{t}} d W_{t}
$$


where $a$ and $c$ are positive constants and $b(\cdot)$ is a non-negative deterministic function. When the mean-reversion function $b$ is constant, the following lemma is a standard result in the affine processes literature (see e.g. [16]). Note that (13), which is obtained from (12) with $y=0$, by differentiating with respect to $t$, gives the conditional density of default time in a classical CIR intensity model.

Lemma 3.1 Consider the process $X$ in (11). If $b(\cdot)$ is constant on $\left[t_{0}, t\right]$, then for every $y \geq 0$,

$$
\begin{gathered}
\mathbb{E}\left(e^{-\int_{t_{0}}^{t} X_{s} d s-y X_{t}} \mid X_{t_{0}}\right)=e^{-\phi\left(t-t_{0} ; y\right) X_{t_{0}}-\xi\left(t-t_{0} ; y\right) b}, \\
\mathbb{E}\left(X_{t} e^{-\int_{t_{0}}^{t} X_{s} d s} \mid X_{t_{0}}\right)=\left(\dot{\phi}\left(t-t_{0} ; 0\right) X_{t_{0}}+\dot{\xi}\left(t-t_{0} ; 0\right) b\right) e^{-\phi\left(t-t_{0} ; 0\right) X_{t_{0}}-\xi\left(t-t_{0} ; 0\right) b},
\end{gathered}
$$

where $\phi$ and $\xi$ satisfy the following Riccati system of ODE:

$$
\left\{\begin{array}{lll}
\dot{\phi}(s ; y)=-a \phi(s ; y)-\frac{c^{2}}{2}(\phi(s ; y))^{2}+1 & ; \quad \phi(0 ; y)=y \\
\dot{\xi}(s ; y)=a \phi(s ; y) & ; \quad \xi(0 ; y)=0 .
\end{array}\right.
$$

Note that the latter ODE can be solved explicitly, i.e.,

$$
\begin{aligned}
\phi(s ; y) & =\frac{1+D(y) e^{-A(y) s}}{B+C(y) e^{-A(y) s}} \\
\xi(s ; y) & =\frac{a}{B}\left\{\frac{C(y)-B D(y)}{A(y) C(y)} \log \frac{B+C(y) e^{-A(y) s}}{B+C(y)}+s\right\} .
\end{aligned}
$$

where $A, B, C$ and $D$ are given by

$$
\begin{aligned}
& B=\frac{1}{2}\left(a+\sqrt{a^{2}+2 c^{2}}\right), \quad C(y)=(1-B y) \frac{a+c^{2} y-\sqrt{a^{2}+2 c^{2}}}{2 a y+c^{2} y-2} \\
& D(y)=(B+C(y)) y-1, \quad A(y)=\frac{-C(y)(2 B-a)+D(y)\left(c^{2}+a B\right)}{B D(y)-C(y)} .
\end{aligned}
$$

In the following proposition, we extend Lemma 3.1 to the case of generalized CIR processes where the mean-reversion function $b(\cdot)$ is assumed to be piecewise constant. We denote $T_{0}=0$. The functions $\phi$ and $\xi$ are those of Lemma 3.1. As a sanity-check observe that when $b_{i}=\cdots=b_{j}$, Proposition 3.2 gives the same results than the ones established in Lemma 3.1 for constant meanreversion CIR processes. Also note that the partial derivative $\partial_{s} I$ of $I$ with respect to $s$ in (18) depends on the functions $\dot{\phi}$ and $\dot{\xi}$, which can be computed explicitly thanks to (14), (15) and (16).

Proposition 3.2 Assume that $b(\cdot)$ is a piecewise constant function: $b(t)=b_{k}$ on $t \in\left[T_{k-1}, T_{k}\right]$ for $k=1, \ldots, m$. For $t<s$, let $i \leq j$ such that $t \in\left[T_{i-1}, T_{i}\right)$ and $s \in\left(T_{j}, T_{j+1}\right]$. Then

(i) For any $y \geq 0$,

$$
\mathbb{E}\left(\exp \left(-\int_{t}^{s} X_{u} d u-y X_{s}\right) \mid X_{t}\right)=\exp \left(-I\left(t, s, X_{t}, y\right)\right)
$$

where

$$
\begin{aligned}
I(t, s, x, y):=x \phi(s-t ; y)+ & b_{i}\left[\xi(s-t ; y)-\xi\left(s-T_{i} ; y\right)\right] \\
& +\sum_{k=i+1}^{j} b_{k}\left(\xi\left(s-T_{k-1} ; y\right)-\xi\left(s-T_{k} ; y\right)\right) \\
& +b_{j+1} \xi\left(s-T_{j} ; y\right) .
\end{aligned}
$$

(ii) One has,

$$
\mathbb{E}\left(X_{s} \exp \left(-\int_{t}^{s} X_{u} d u\right) \mid X_{t}\right)=\partial_{s} I\left(t, s, X_{t}, 0\right) \mathbb{E}\left(\exp \left(-\int_{t}^{s} X_{u} d u\right) \mid X_{t}\right) .
$$


Proof. Expression (18) can be obtained by differentiating (17) with respect to $s$ and by letting $y=0$. Let us prove (17). Note that the piecewise constant function $b(\cdot)$ is characterized by the time tenor $\boldsymbol{T}=\left(T_{k}\right)_{k=1, \ldots, m}$ and the corresponding set of parameters $\boldsymbol{b}=\left(b_{k}\right)_{k=1, \ldots, m}$. From Prop. 8.2 in [7, we know that when $t<s$ such that $t \in\left[T_{i-1}, T_{i}\right)$ and $s \in\left(T_{j}, T_{j+1}\right]$ with $i \leq j$, the following relation holds

$$
\begin{aligned}
& \mathcal{E}(\boldsymbol{T}, \boldsymbol{b}, x, y):=\mathbb{E}\left(\exp \left(-\int_{t}^{s} X_{u} d u-y X_{s}\right) \mid X_{t}=x\right)= \\
& \quad \exp \left\{-x \phi\left(T_{i}-t ; y_{i}(s)\right)-b_{i} \xi\left(T_{i}-t ; y_{i}(s)\right)-\sum_{k=i+1}^{j} b_{k} \xi\left(T_{k}-T_{k-1} ; y_{k}(s)\right)-b_{j+1} \xi\left(s-T_{j} ; y\right)\right\}
\end{aligned}
$$

where the $y_{k}(\mathrm{~s}), k=i, \ldots, j$, are defined by the following backward recursion

$$
\begin{aligned}
& y_{j}(s):=\phi\left(s-T_{j} ; y\right), \\
& y_{k}(s):=\phi\left(T_{k+1}-T_{k} ; y_{k+1}(s)\right), \quad k<j .
\end{aligned}
$$

Note that in the particular case where $b_{j}=b_{j+1}$, expression (19) must be equal to the one obtained when $T_{j}$ is removed from the set $\boldsymbol{T}$ and $b_{j}$ is removed from the set $\boldsymbol{b}$. More precisely, if the adjusted set of parameters are defined by $\tilde{\boldsymbol{T}}:=\boldsymbol{T} \backslash\left\{T_{j}\right\}$ and $\tilde{\boldsymbol{b}}:=\boldsymbol{b} \backslash\left\{b_{j}\right\}$ then, the following relation holds

$$
\left.\mathcal{E}(\boldsymbol{T}, \boldsymbol{b}, x, y)\right|_{b_{j}=b_{j+1}}=\mathcal{E}(\tilde{\boldsymbol{T}}, \tilde{\boldsymbol{b}}, x, y) .
$$

By letting $x, b_{i}, \ldots, b_{j-1}$ be equal to zero in the previous relation, we obtain

$$
\xi\left(T_{j}-T_{j-1} ; y_{j}(s)\right)=\xi\left(s-T_{j-1} ; y\right)-\xi\left(s-T_{j} ; y\right) .
$$

We can then replace $\xi\left(T_{j}-T_{j-1} ; y_{j}(s)\right)$ in (19) by the right hand side of (22) and apply the previous argument in the particular case where $b_{j-1}=b_{j}=b_{j+1}$. We then obtain

$$
\xi\left(T_{j-1}-T_{j-2} ; y_{j-1}(s)\right)=\xi\left(s-T_{j-2} ; y\right)-\xi\left(s-T_{j-1} ; y\right) .
$$

This reasoning can be done backward from $k=j-2$ to $k=i$ which shows that

$$
\xi\left(T_{k}-T_{k-1} ; y_{k}(s)\right)=\xi\left(s-T_{k-1} ; y\right)-\xi\left(s-T_{k} ; y\right)
$$

and

$$
\xi\left(T_{i}-t ; y_{i}(s)\right)=\xi(s-t ; y)-\xi\left(s-T_{i} ; y\right) .
$$

Eventually, in the particular case where $b_{i}=\cdots=b_{j+1}$, expression (19) must be the same as the one given by application of Lemma 3.1, i.e.,

$$
\mathcal{E}(\boldsymbol{T}, \boldsymbol{b}, x, y)=\exp \left(-x \phi(s-t ; y)-b_{i} \xi(s-t ; y)\right) .
$$

So, by letting $b_{i}$ equal to 0 , we obtain $\phi\left(T_{i}-t ; y_{i}(s)\right)=\phi(s-t ; y)$ which concludes the proof.

Remark 3.3 Proposition 3.2 could also be used to estimate survival probabilities or conditional densities when the mean-reversion function $b(\cdot)$ is more complex (non necessarily piecewise constant). Indeed, we could look for a good piecewise-constant approximation of $b(\cdot)$ and then apply Proposition 3.2 to it.

\subsection{CDS Pricing}

We assume in the sequel that recovery rates are independent of default times. Under this assumption, the CDS spread of a particular name can be expressed as deterministic functions of its survival probabilities and of its expected recovery. Let $t_{1}<\cdots<t_{p}=T$ be the remaining premium payment dates where $T$ stands for the maturity date. We assume for simplicity that the risk-free 
interest rate is constant and equal to $r$ and we denote $\beta(t)=e^{-r t}$ the corresponding discount factor. In our numerical experiment, the current fair CDS spread of name $i$ is approximated by the following expression

$$
S_{i}(T)=\left(1-R_{i}^{*}\right) \frac{\sum_{j=1}^{p} \beta\left(t_{j}\right)\left(\mathbb{P}\left(\tau_{i}>t_{j-1}\right)-\mathbb{P}\left(\tau_{i}>t_{j}\right)\right)}{\sum_{j=1}^{p} \beta\left(t_{j}\right)\left(t_{j}-t_{j-1}\right) \mathbb{P}\left(\tau_{i}>t_{j}\right)}
$$

where $R_{i}^{*}$ denotes the expected recovery rate of name $i$. To derive the previous expression, we implicitly assume that, if a default occur at time $\tau_{i}<T$, the protection payment occurs at the premium payment date that immediately follows $\tau_{i}$.

Recall that (cf. (9))

$$
\mathbb{P}\left(\tau_{i}>t_{j}\right)=\mathbb{E} \exp \left(-\int_{0}^{t_{j}} X_{s}^{i} d s\right)
$$

where $X^{i}$ is an extended CIR process with parameters $a, c$ and piecewise-constant mean-reversion function $b_{i}(\cdot)$ in (5) (assuming piecewise-constant functions $b_{Y}(\cdot)$ ). Hence, provided the expected recovery is known, the CDS spread of name $i$ can be efficiently calculated using part $(i)$ of Proposition 3.2 with $y=0$.

\subsection{CDO Tranche Pricing with Random Recoveries}

In this subsection we outline how to modify the Markov copula to include possibly random but independent recoveries, giving numerical details in complement of the brief outline in [4]. Stochastic recoveries do not break the Markovian nor the Markovian copula structure. However by introducing random recoveries we can no longer use exact convolution recursion procedures for pricing CDO tranches. As briefly outlined in [4] one can instead use an approximate procedure based on the exponential approximations of the so called hockey stick function of [3, 19, 20]. In this subsection we explain in detail how to use this method for computing the price of a CDO tranche in our Markov model when the individual losses are random.

The mathematical ideas underlying the method of exponential approximations were originally developed by [3], and was later adopted by Iscoe et al. in [19] and 20] to price CDO tranches in a Gaussian-copula model. While [19], 20] uses constant recoveries, we will in this paper adopt their techniques to random recoveries. Below we will outline the techniques given in [19], [20] and our presentation also introduces notation needed later on. First, recall that the portfolio credit loss $L_{t}$ at time $t$ is defined as $L_{t}:=\sum_{i}\left(1-R_{i}\right) H_{t}^{i}$ where the variables $R_{i}$ are random and independent fractional recoveries with values in $[0,1]$. Next, the so called tranche-loss function $L_{t}^{a, b}$ for the tranche $[a, b]$ as a function of the portfolio credit loss $L_{t}$ is given by

$$
L_{t}^{a, b}=\left(L_{t}-a\right)^{+}-\left(L_{t}-b\right)^{+}
$$

where $x^{+}=\max (x, 0)$, see also Figure 1 .

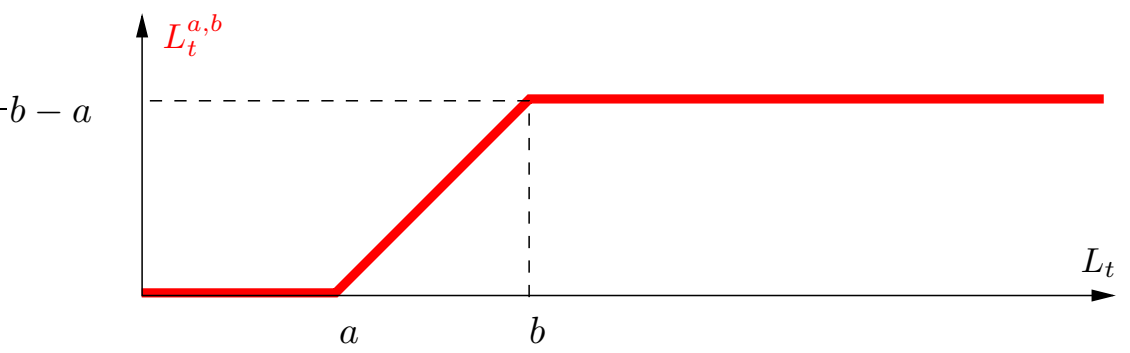

Figure 1: The tranche loss for $[a, b]$ as function of the total loss $L_{t}$. 
Furthermore, as in [3], we introduce the so-called hockey stick function $h(x)$ given by

$$
h(x)= \begin{cases}1-x & \text { if } 0 \leq x \leq 1 \\ 0 & \text { if } 1<x\end{cases}
$$

see also in Figure 2

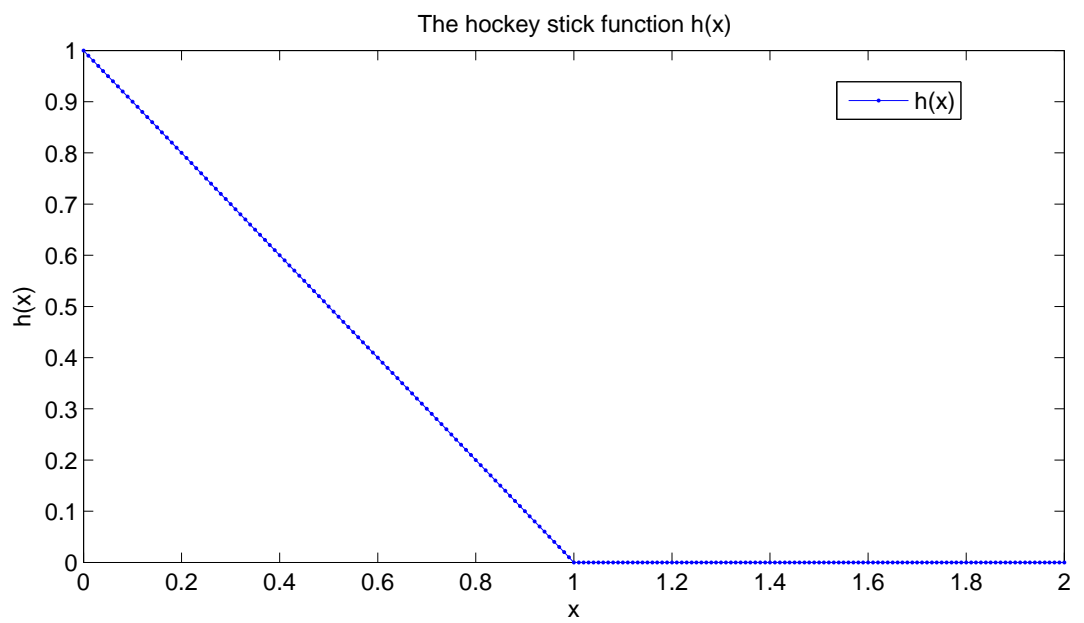

Figure 2: The hockey stick function $h(x)$ for $x \in[0,2]$.

Let $c>0$ be scalar. By using (29) one can show that

$$
\min (x, c)=c-\operatorname{ch}\left(\frac{x}{c}\right)
$$

and for any two scalars $a$ and $b$ it holds that

$$
(x-a)^{+}-(x-b)^{+}=\min (x, b)-\min (x, a)
$$

so (30) and (31) then yields

$$
(x-a)^{+}-(x-b)^{+}=b\left(1-h\left(\frac{x}{b}\right)\right)-a\left(1-h\left(\frac{x}{a}\right)\right) .
$$

Hence, (28) and (32) implies that

$$
L_{t}^{a, b}=b\left(1-h\left(\frac{L_{t}}{b}\right)\right)-a\left(1-h\left(\frac{L_{t}}{a}\right)\right)
$$

and this observations was done by [19] which combined (33) with the results of [3]. More specifically, [3] shows that for any fixed $\epsilon>0$, the function $h(x)$ can be approximated by a function $h_{\exp }^{(q)}(x)$ on $[0, d]$ with $d=d(\epsilon)$ so that $\left|h(x)-h_{\exp }^{(q)}(x)\right| \leq \epsilon$ for all $x \in[0, d]$ where $q=q(\epsilon)$ is a positive integer and $h_{\exp }^{(q)}(x)$ is given by

$$
h_{\exp }^{(q)}(x)=\sum_{\ell=1}^{q} \omega_{\ell} \exp \left(\gamma_{\ell} \frac{x}{d}\right) .
$$

Here $\left(\omega_{\ell}\right)_{\ell=1}^{q}$ and $\left(\gamma_{\ell}\right)_{\ell=1}^{q}$ are complex numbers obtained as roots of polynomials whose coefficients can be computed numerically in a straightforward way. Figure 3 visualizes the approximation $h_{\exp }^{(q)}(x)$ of $h(x)$ on $x \in[0,10]$ for $q=2,5,10$ and $q=50$. As can be seen in Figure 3, the approximation is fairly good already for small values values of $q$. In [19] the authors choose the algorithm for computing 
$\left(\omega_{\ell}\right)_{\ell=1}^{q}$ and $\left(\gamma_{\ell}\right)_{\ell=1}^{q}$ so that $d(\epsilon)=2$, and then they show that the approximation accuracy $\epsilon$ satisfies $\frac{1}{4(q+1)} \leq \epsilon$ where $q$ are the number terms in (34). Thus, $q$ can be chosen first, implying an accuracy $\epsilon$ so that $\frac{1}{4(q+1)} \leq \epsilon$. In practice, the error $\left|h(x)-h_{\exp }^{(q)}(x)\right|$ will for almost all $x \in[0, \infty)$ be much smaller than the lower bound $\frac{1}{4(q+1)}$ for $\epsilon$, as can bee seen in Figure 4 More specifically, in [19], [20] the authors show that $\operatorname{Re}\left(\gamma_{\ell}\right)<0$ for all $\ell$ (see also in Figure 5) which implies that $h_{\exp }^{(q)}(x) \rightarrow 0$ as $x \rightarrow \infty$ and, as pointed out by [19], since $h(x)=0$ for $x \geq 1$ this guarantees that $h_{\exp }^{(q)}(x) \rightarrow h(x)$ when $x \rightarrow \infty$. In the rest of this paper we will, just as in [19, 20, 4], use $d=2$ in the approximation $h_{\exp }^{(q)}(x)$ given by (34).
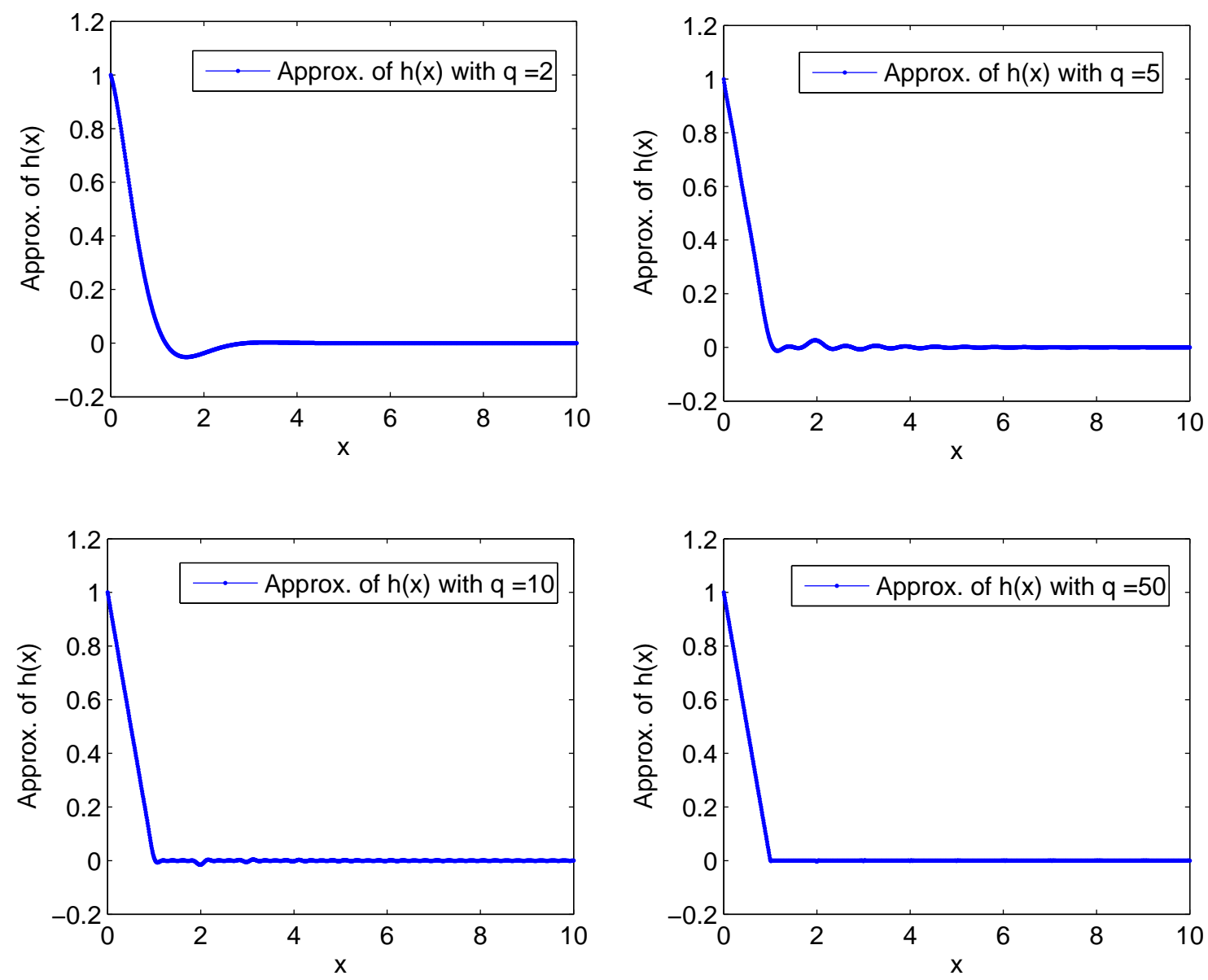

Figure 3: The function $h_{\exp }^{(q)}(x)$ as approximation of $h(x)$ for $x \in[0,10]$ with $q=2,5,10$ and $q=50$.

Since $\left(\omega_{\ell}\right)_{\ell=1}^{q}$ are roots to a certain polynomial, then if $\zeta \in\left(\omega_{\ell}\right)_{\ell=1}^{q}$ it will also hold that $\bar{\zeta} \in\left(\omega_{\ell}\right)_{\ell=1}^{q}$. The same also holds for the complex numbers $\left(\gamma_{\ell}\right)_{\ell=1}^{q}$. Thus, it will hold that $\operatorname{Im}\left(\sum_{\ell=1}^{q} \gamma_{\ell} \exp \left(\gamma_{\ell} x / d\right)\right)=0$ and Figure 5 displays the coefficients $\left(\omega_{\ell}\right)_{\ell=1}^{q}$ and $\left(\gamma_{\ell}\right)_{\ell=1}^{q}$ in the case $q=50$. 

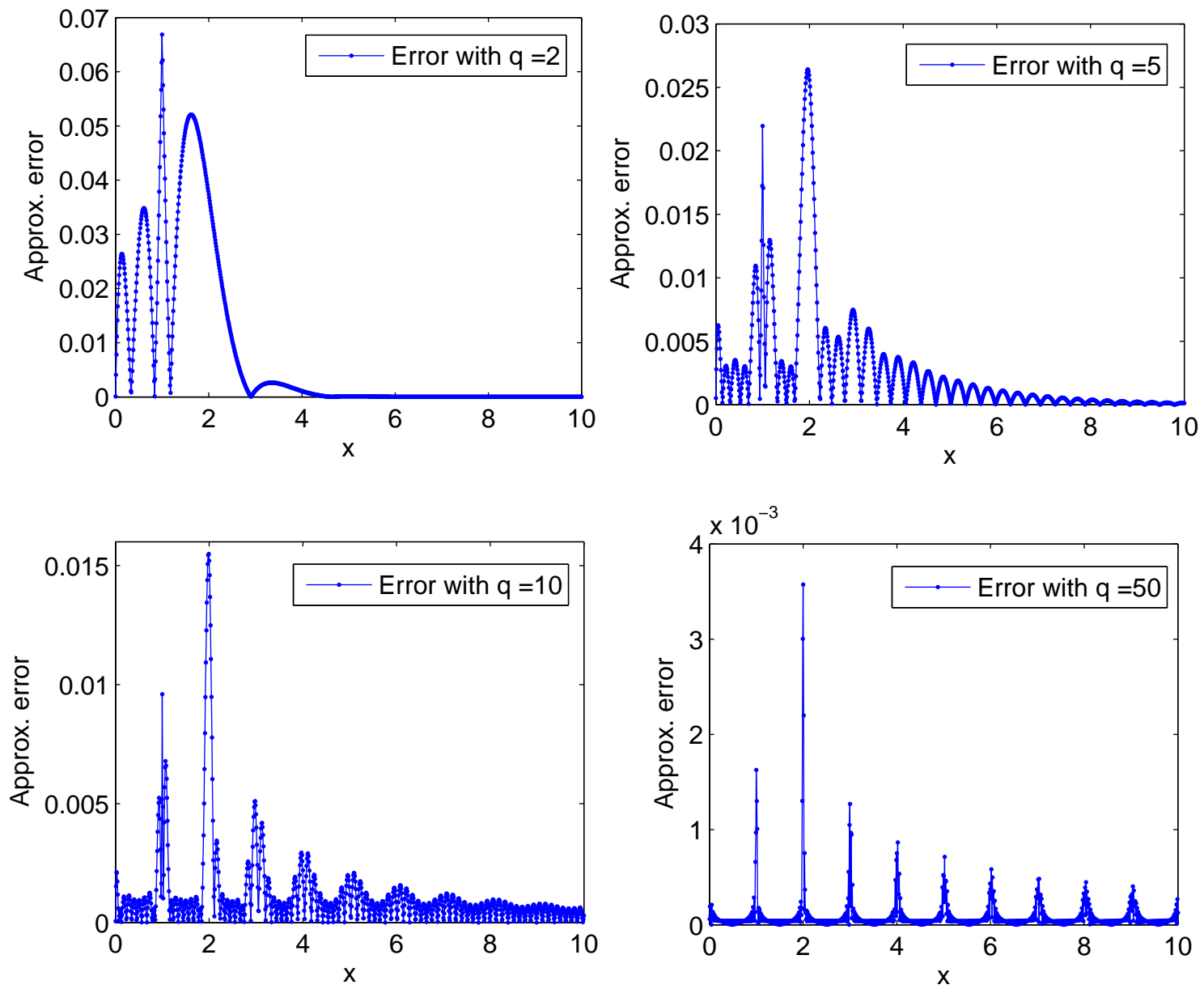

Figure 4: The approximation error $\left|h(x)-h_{\exp }^{(q)}(x)\right|$ for $x \in[0,10]$ with $q=2,5,10$ and $q=50$.

It is well known that in order to price synthetic CDO tranches, one needs to compute the quantity $\mathbb{E}\left[L_{t}^{a, b}\right]$ for $t>0$, see e.g. in [18]. So by replacing $h(x)$ with $h_{\exp }^{(q)}(x)$ in (33) with $d=2$ and using (34) then implies that we can approximate $L_{t}^{a, b}$ as follows

$$
L_{t}^{a, b} \approx b-a-b \sum_{\ell=1}^{q} \omega_{\ell} \exp \left(\gamma_{\ell} \frac{L_{t}}{2 b}\right)+a \sum_{\ell=1}^{q} \omega_{\ell} \exp \left(\gamma_{\ell} \frac{L_{t}}{2 a}\right)
$$

and consequently

$$
\mathbb{E}\left[L_{t}^{a, b}\right] \approx b-a-b \sum_{\ell=1}^{q} \omega_{\ell} \mathbb{E}\left[\exp \left(\gamma_{\ell} \frac{L_{t}}{2 b}\right)\right]+a \sum_{\ell=1}^{q} \omega_{\ell} \mathbb{E}\left[\exp \left(\gamma_{\ell} \frac{L_{t}}{2 a}\right)\right]
$$


The $\gamma_{1}$ coefficients for $l=1,2, \ldots, q$ when $q=50$
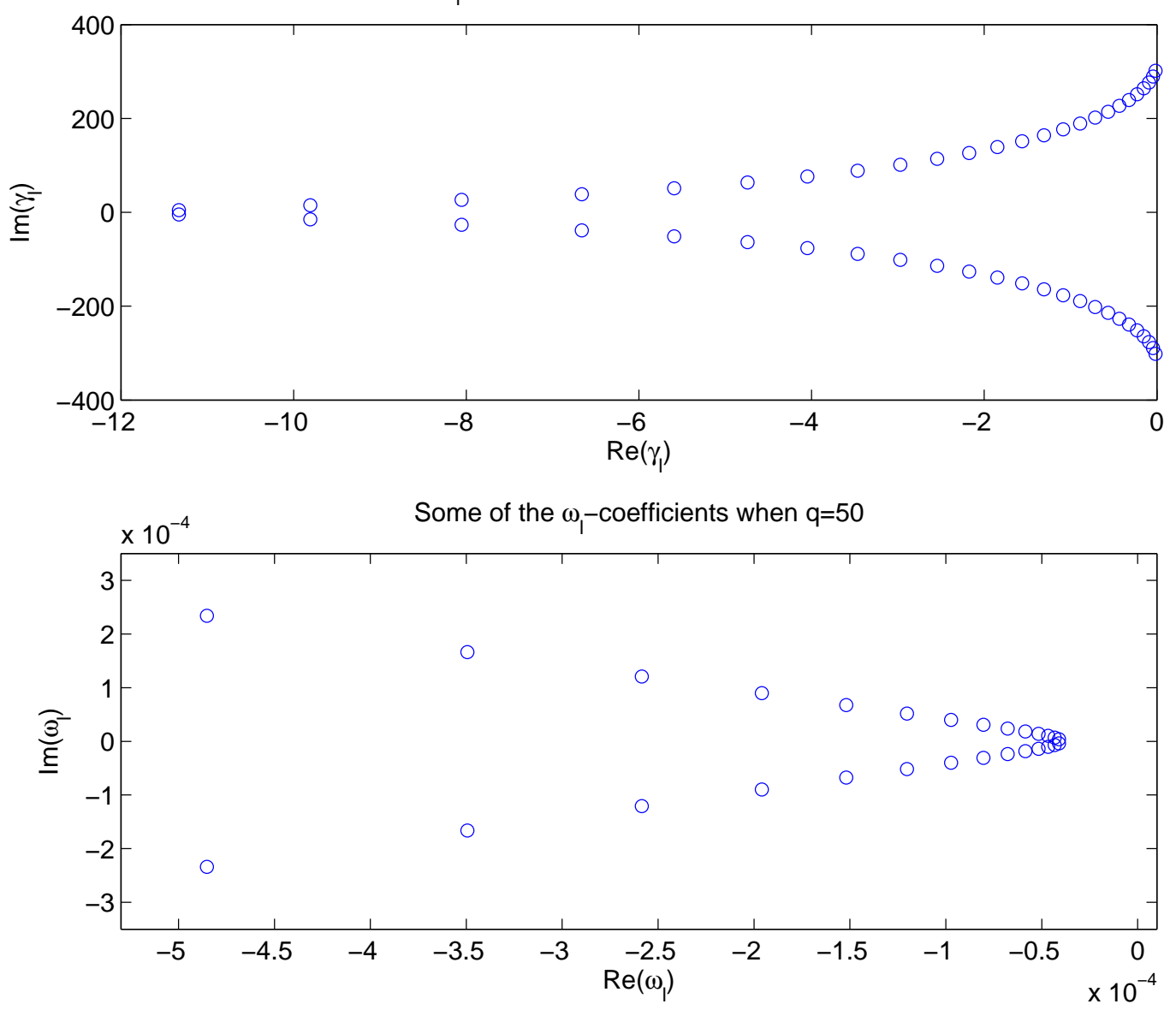

Figure 5: The coefficients $\left(\gamma_{\ell}\right)_{\ell=1}^{q}$ (top) and some of the coefficients $\left(\omega_{\ell}\right)_{\ell=1}^{q}$ (bottom) when $q=50$.

Thus, in view of (36), the pricing of a CDO tranche of maturity $T$, boils down to computation of expectations of the form

$$
\mathbb{E} e^{\gamma_{\ell} \frac{L_{t}}{2 c}}
$$

for $\ell=1,2, \ldots, q$ and different attachment points $c$ and time horizons $0 \leq t \leq T$.

Remark 3.4 One can extend the present developments to conditional expectations given $\mathcal{F}_{s}$ for any $0<s<t$. The case $s=0$ is used in the calibration (our focus in this paper), while the case $s>0$ is needed for pricing the credit valuation adjustment (CVA) on a CDO tranche in a counterparty risky environment, a topical issue since the 2007-09 credit crisis (see [15]).

Since the algorithm for computing $\mathbb{E} e^{\gamma_{\ell} \frac{L_{t}}{2 c}}$ is the same for each $\ell=1,2, \ldots, q$ and any attachment point $c$, we will below for notational convenience simply write $\mathbb{E} e^{\gamma L_{t}}$ instead of $\mathbb{E} e^{\gamma_{\ell} \frac{L_{t}}{2 c}}$

We now use the common shock model representation developed in Subsection 2.3 of [4, with the same notation that was introduced there except that $\theta$ there is $t$ here, and $t$ there is simply 0 here, as we focus here on expectations and not conditional expectations; moreover we now use a "थ" notation for the common shocks model representation at the starting time 0 below, instead of a 
" $(t)$ " which is used for common shocks model representation with a varying forward starting time $t$ in [4]. $Y \in \mathcal{Y}$

We thus introduce a common shocks copula model of default times $\widehat{\tau}_{Y}$ defined by, for every

$$
\widehat{\tau}_{Y}=\inf \left\{t>0 ; \int_{0}^{t} X_{s}^{Y} d s>E_{Y}\right\}
$$

where the random variables $E_{Y}$ are i.i.d. and exponentially distributed with parameter 1. For every obligor $i$ we let

$$
\widehat{\tau}_{i}=\min _{\{Y \in \mathcal{Y} ; i \in Y\}} \widehat{\tau}_{Y},
$$

which defines the default time of obligor $i$ in the common shocks copula model. We also introduce the indicator processes $\widehat{H}_{t}^{Y}=\mathbb{1}_{\left\{\widehat{\tau}_{Y} \leq t\right\}}$ and $\widehat{H}_{t}^{i}=\mathbb{1}_{\left\{\widehat{\tau}_{i} \leq t\right\}}$, for every triggering-event $Y$ and obligor $i$. One then has much like in Proposition 2.10(ii) of [4] that

$$
\mathbb{E} e^{\gamma L_{t}}=\mathbb{E} e^{\gamma \widehat{L}_{t}}
$$

where $\widehat{L}_{t}:=\sum_{i}\left(1-R_{i}\right) \widehat{H}_{t}^{i}$.

We henceforth assume a nested structure of the sets $I_{j}$ given by

$$
I_{1} \subset \ldots \subset I_{m} .
$$

This structure implies that if all obligors in group $I_{k}$ have defaulted, then all obligors in group $I_{1}, \ldots, I_{k-1}$ have also defaulted. As detailed in [4], the nested structure (40) yields a particularly tractable expression for the portfolio loss distribution. This nested structure also makes sense financially with regards to the hierarchical structure of risks which is reflected in standard CDO tranches. Denoting conventionally $I_{0}=\emptyset$ and $\widehat{H}_{t}^{I_{0}}=1$, then the event-sets

$$
\widehat{\Omega}_{t}^{j}:=\left\{\widehat{H}_{t}^{I_{j}}=1, \widehat{H}_{t}^{I_{j+1}}=0, \ldots, \widehat{H}_{t}^{I_{m}}=0\right\}, 0 \leq j \leq m
$$

form a partition of $\Omega$ with

$$
\mathbb{P}\left(\widehat{\Omega}_{t}^{j}\right)=\left(1-\mathbb{E} e^{-\int_{0}^{t} X_{s}^{I_{j}} d s}\right) \prod_{j+1 \leq l \leq m} \mathbb{E} e^{-\int_{0}^{t} X_{s}^{I_{l}} d s}
$$

where the expectations are explicitly given by Proposition 3.2(i). One then has in (39) that

$$
\mathbb{E} e^{\gamma \widehat{L}_{t}}=\sum_{0 \leq j \leq m} \mathbb{E}\left(e^{\gamma \widehat{L}_{t}} \mid \widehat{\Omega}_{t}^{j}\right) \mathbb{P}\left(\widehat{\Omega}_{t}^{j}\right)
$$

in which by conditional independence of the $\widehat{H}_{t}^{i}$ given every $\widehat{\Omega}_{t}^{j}$

$$
\mathbb{E}\left(e^{\gamma \widehat{L}_{t}} \mid \widehat{\Omega}_{t}^{j}\right)=\mathbb{E}\left(e^{\gamma \sum_{i}\left(1-R_{i}\right) \widehat{H}_{t}^{i}} \mid \widehat{\Omega}_{t}^{j}\right)=\prod_{i \in Z} \mathbb{E}\left(e^{\gamma\left(1-R_{i}\right) \widehat{H}_{t}^{i}} \mid \widehat{\Omega}_{t}^{j}\right) .
$$

Now observe that by independence of $R_{i}$

$$
\mathbb{E}\left(e^{\gamma\left(1-R_{i}\right) \widehat{H}_{t}^{i}} \mid \widehat{\Omega}_{t}^{j}\right)= \begin{cases}\mathbb{E} e^{\gamma\left(1-R_{i}\right)}, & i \in I_{j} \\ \mathbb{E} e^{\gamma\left(1-R_{i}\right) \widehat{H}_{t}^{\{i\}}}, & \text { else }\end{cases}
$$

with

$$
\mathbb{E} e^{\gamma\left(1-R_{i}\right) \hat{H}_{t}^{\{i\}}}=1-\widehat{p}_{t}^{i, j}\left(1-\mathbb{E} e^{\gamma\left(1-R_{i}\right)}\right)
$$

where

$$
\widehat{p}_{t}^{i, j}= \begin{cases}1, & i \in I_{j} \\ 1-\mathbb{E} e^{-\int_{0}^{t} X_{s}^{\{i\}} d s} & \text { else }\end{cases}
$$


in which the expectation is explicitly given by Proposition 3.2(i). Hence, the above formulas together with Equation (39) will determine the quantity (37) which in turn is needed to compute the expected tranche loss given by Equation (36). Furthermore, from the above equations we see that what is left to compute is the quantity $\mathbb{E} e^{\gamma\left(1-R_{i}\right)}$ and in Subsection 4.2 we will give an explicitly example of the recovery rate $R_{i}$ (and the quantity $\mathbb{E} e^{\gamma\left(1-R_{i}\right)}$ ) which was used in [4] with the hockey-stick methodwith the above hockey-stick method when calibrating the Markov copula against market data on CDO tranches. As seen in [4], using random recoveries will for some data sets render much better calibration results compared with the case of using constant recoveries.

\section{Calibration}

\subsection{Stochastic Intensity and Constant Recovery}

In this subsection, we discuss in more details the calibration methodology that has been used in [4] when fitting the stochastic intensity Markov copula model against CDO tranches on CDX.NA.IG series. We use here extended CIR intensities with piecewise-constant mean-reversion coefficients (as described previously) and we assume that recovery rates are constant. In Subsection4.2 we will investigate the "dual" model specification where intensities are deterministic and recoveries are stochastic.

Recall that, given non-negative constants $a$ and $c$, the intensity process of any group $Y \in \mathcal{Y}$ is defined by

$$
d X_{t}^{Y}=a\left(b_{Y}(t)-X_{t}^{Y}\right) d t+c \sqrt{X_{t}^{Y}} d W_{t}^{Y}
$$

where $X_{0}^{Y}$ is a given constant and $b_{Y}(t)$ is a piecewise constant function such that, for every $k=$ $1 \ldots M, b_{Y}(t)=b_{Y}^{(k)}, t \in\left[T_{k-1}, T_{k}\right)$ with $T_{0}=0$. In this paper we will use a time tenor consisting of two maturities $T_{1}=3 y$ and $T_{2}=5 y$. Moreover, in order to reduce the number of parameters at hands, we consider that, for every group $Y \in \mathcal{Y}$, the starting point of the corresponding intensity process is given by its first-pillar mean-reversion parameter, i.e., $X_{0}^{Y}=b_{Y}^{(1)}$. Note that in that case, given $a$ and $c$, the intensity dynamics of any group $Y \in \mathcal{Y}$ is completely characterized by $b_{Y}^{(k)}$, $k=1,2$. In particular, thanks to (4), the survival probability of name $i$ up to $T_{2}$ is characterized by $\left(b_{i}^{(k)}\right)_{k=1,2}$ where

$$
b_{i}^{(k)}=b_{\{i\}}^{(k)}+\sum_{\mathcal{I} \ni I \ni i} b_{I}^{(k)} .
$$

The calibration is done in two steps. The first step consists in boostrapping $\left(b_{i}^{(k)}\right)_{k=1,2}$ on the single-name CDS curve associated with obligor $i$, for any $i=1, \ldots, n$. The CDS curve of name $i$ is composed of two market spreads: $S_{i}^{*}\left(T_{1}\right)$ corresponding to maturity $T_{1}$ and $S_{i}^{*}\left(T_{2}\right)$ corresponding to maturity $T_{2}$. We first remark from (26) and Proposition 3.2 that the model spread of CDS $i$ with maturity $T_{1}$ only depends on $b_{i}^{(1)}$ whereas the model spread of CDS $i$ with maturity $T_{2}$ depends on $b_{i}^{(1)}$ and $b_{i}^{(2)}$. As soon as $a$ and $c$ are fixed, we can then find $b_{1}^{(1)}$ as the solution of the non-linear (univariate) equation $S_{i}\left(T_{1}\right)=S_{i}^{*}\left(T_{1}\right)$, plugged this solution into the expression of $S_{i}\left(T_{2}\right)$ and then find $b_{1}^{(2)}$ as the solution of the non-linear (univariate) equation $S_{i}\left(T_{2}\right)=S_{i}^{*}\left(T_{2}\right)$. Figure 6 and 7 respectively show the $3 \mathrm{y}$ - and the 5 y-implied mean-reversion coefficients bootstrapped from the 125 CDS curves of the CDX.NA.IG index constituents as of December 17, 2007. We compare three different specifications of the underlying individual intensities : piecewise-constant deterministic intensities (standard bootstrap procedure), CIR intensities with $a=3$ and $c=0.05$ and CIR intensities with $a=3$ and $c=2$. We can see that the volatility parameter $c$ has little impact on implied coefficients whereas individual intensities may be relatively volatile even for small volatility parameter as illustrated by Figure 8 


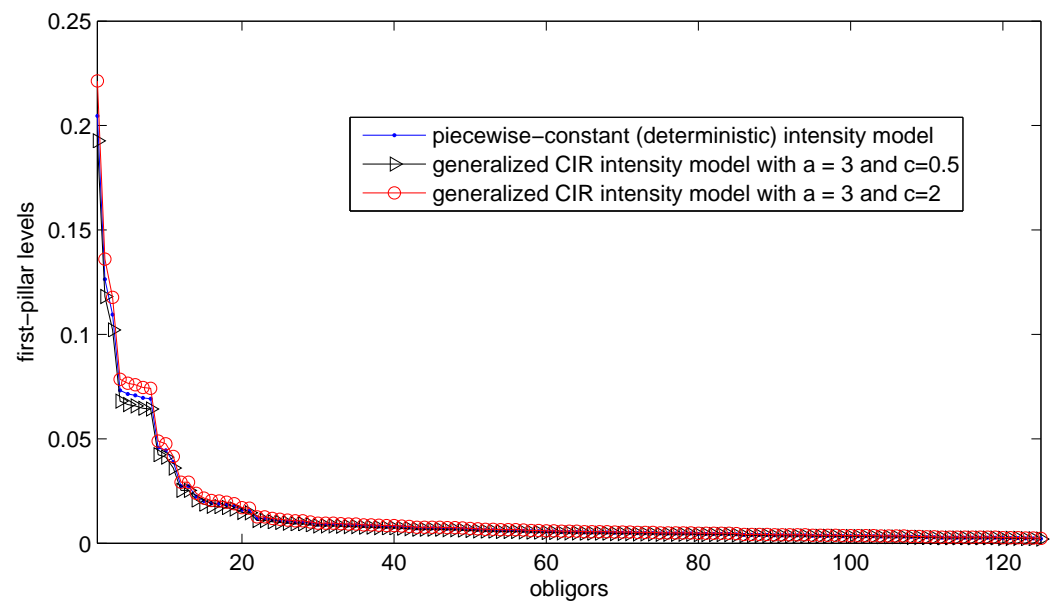

Figure 6: 3-year mean-reversion coefficients $b_{i}^{(1)}, i=1, \ldots, 125$ bootstrapped from CDX.NA.IG December 17, 2007 single-name CDS curves and sorted in decreasing order.

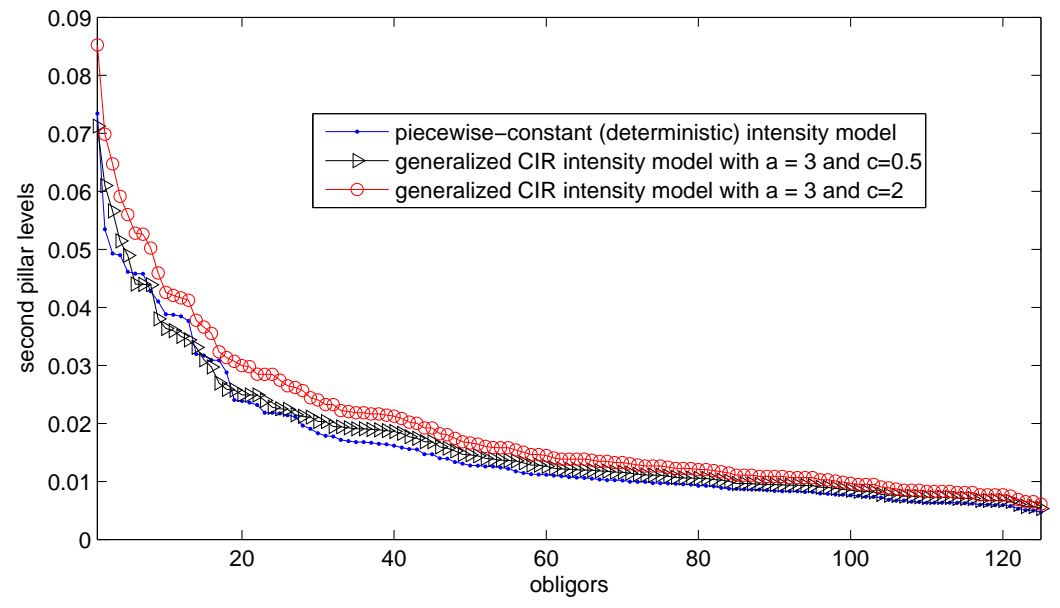

Figure 7: 5-year mean-reversion coefficients $b_{i}^{(2)}, i=1, \ldots, 125$ bootstrapped from CDX.NA.IG December 17, 2007 single-name CDS curves and sorted in decreasing order. 


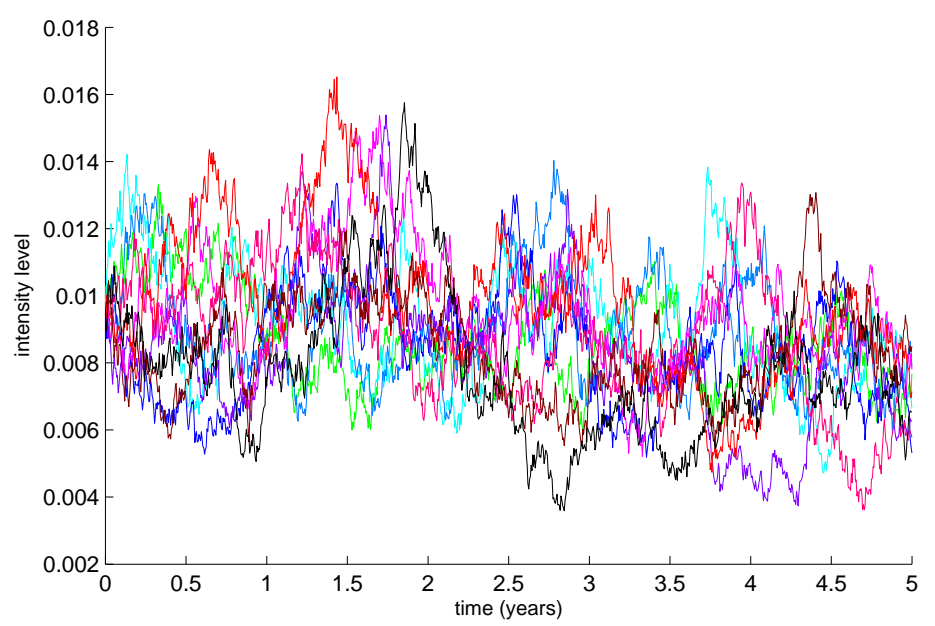

Figure 8: Sample paths of generalized CIR intensities with $a=3$ and $c=0.05$ where meanreversion parameters are implied from AIG CDS curve at December 17, 2007. The first and the second pillar coefficients are (resp.) equal to $b^{(1)}=0.096$ and $b^{(2)}=0.075$.

The second step is to calibrate group parameters $\left(b_{I_{j}}^{(k)}\right)_{k=1,2}, j=1, \ldots, m$ so that the model CDO tranche spreads coincide with the corresponding market spreads. The hockey-stick method described in Subsection 3.3 can be used to compute model CDO tranche spreads.

Moreover, in view of (45), we impose that, for all $k=1,2$ and $i=1, \ldots, n$, the group parameters are such that

$$
\sum_{\mathcal{I} \ni I \ni i} b_{I}^{(k)} \leq b_{i}^{(k)}
$$

for all $i=1, \ldots, 125$. The previous constraints guarantee that the long-term averages $b_{\{i\}}^{(k)}$ of singlegroup intensities are all positive. This in turn implies by construction that the starting points of single-group intensities $X_{0}^{\{i\}}$ are all positive. Given the nested structure of the groups $I_{j}$-s specified in (40), the following constrains must hold for all $l=1, \ldots, m$ and $k=1,2$ :

$$
\sum_{j=l}^{m} b_{I_{j}}^{(k)} \leq \min _{i \in I_{l} \backslash I_{l-1}} b_{i}^{(k)}
$$

Next, the group parameters $\boldsymbol{b}=\left(b_{I_{j}}^{(k)}\right)_{j, k}=\left\{b_{I_{j}}^{(k)}: j=1, \ldots, m\right.$ and $\left.k=1,2\right\}$ are then calibrated so that the five-year model spread $S_{a_{l}, b_{l}}(\boldsymbol{\lambda})=: S_{l}(\boldsymbol{\lambda})$ will coincide with the corresponding market spread $S_{l}^{*}$ for each tranche $l$. To be more specific, the parameters $\boldsymbol{b}=\left(b_{I_{j}}^{(k)}\right)_{j, k}$ are obtained according to

$$
\boldsymbol{b}=\underset{\widehat{\boldsymbol{b}}}{\operatorname{argmin}} \sum_{l}\left(\frac{S_{l}(\widehat{\boldsymbol{b}})-S_{l}^{*}}{S_{l}^{*}}\right)^{2}
$$

under the constraints that all elements in $\boldsymbol{b}$ are nonnegative and that $\boldsymbol{b}$ satisfies the inequalities (47). In $S_{l}(\widehat{\boldsymbol{b}})$ we have emphasized that the model spread for tranche $l$ is a function of $\boldsymbol{b}=\left(b_{I_{j}}^{(k)}\right)_{j, k}$ but we suppressed the dependence in other parameters like interest rate, payment frequency or $b_{i}$, $i=1, \ldots, n$. In the calibration we used an interest rate of $3 \%$, the payments in the premium leg were quarterly and the integral in the default leg was discretized on a quarterly mesh. We use a constant recovery of 40\%. The calibration results on CDX.NA.IG December 17, 2007 market quotes can be found in 4 . 
As a matter of comparison, we plot in Figure 9 the loss distribution functions obtained from fitted parameters of the generalized CIR intensity model with $a=3$ and $c=0.5$ and from the fitted parameters of the piecewise-constant (deterministic) intensity model. Note that the grouping is not the same in the two calibrated models. For the deterministic intensity model, we used 5 groups $I_{1}, I_{2}, \ldots, I_{5}$ where $I_{j}=\left\{1, \ldots, i_{j}\right\}$ for $i_{j}=6,19,25,61,125$ when calibrating the joint default intensities. However, for the two cases, we label the obligors by decreasing level of riskiness. We use the average over 3-year and 5-year CDS spreads as a measure of riskiness. Consequently, obligor 1 has the highest average CDS spread while company 125 has the lowest average CDS spread. Moreover, the obligors in the set $I_{5} \backslash I_{4}$ consisting of the 64 safest companies are assumed to never default individually, and the corresponding CDSs are excluded from the calibrations constraints. This specification renders a perfect fit. For the CIR intensity model, we also use 5 groups but with $i_{j}=8,19,27,102,125$ and, contrary to the deterministic intensity model, we do not remove any name from the calibration constraints. This specification renders a very good fit (see [4] for more details).

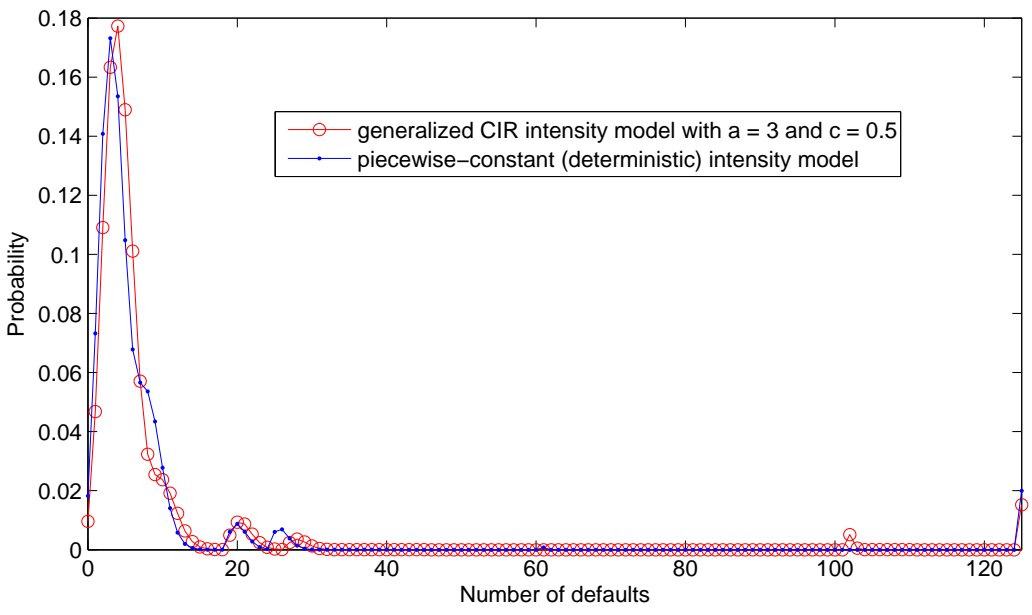

Figure 9: Comparison of 5-year implied loss distributions $\left(\mathbb{P}\left(\sum_{i} H_{t}^{i}=k\right), k=0, \ldots, 125\right)$ from CDX.NA.IG December 17, 2007 calibration of the generalized CIR intensity model and the piecewise constant intensity model.

\subsection{Binomially Distributed Random Recoveries}

In this subsection we describe and discuss the distribution for the individual random recoveries, a binomial mixture distribution, which was used in [4] with the hockey-stick method and piecewise constant default intensities, when calibrating the Markov copula against market data on CDO tranches. As seen in [4, using this type of random recoveries will for some data sets render much better calibration results compared with the case of using constant recoveries. In particular, we will in this subsection give a detailed derivation of the accompanying constraints which in [4] was added to the calibration of the CDO tranches simultaneously with the other constraints for the common shock intensities, in the case with piecewise constant default intensities. This constraint for the parameters connected to the random recovery was stated in [4 without a proof. We however remark that the outline in this subsection is independent of the type of default intensities that are used for the groups and individual names.

We assume that the individual recoveries $\left\{R_{i}\right\}$ are i.i.d and have a binomial mixture distribution on the following form

$$
R_{i} \sim \frac{1}{K} \operatorname{Bin}\left(K, R^{*}\left(p_{0}+(1-\Theta) p_{1}\right)\right) \text { where } \Theta \in\{0,1\} \text { and } \mathbb{P}[\Theta=1]=q
$$


where $R^{*}, q p_{0}$ and $p_{1}$ are positive constants and $K$ is an integer (in this paper and in [4] we let $K=10)$. As a result, the distribution function for the recovery rate is given by

$$
\mathbb{P}\left[R_{i}=\frac{k}{K}\right]=\sum_{\xi=0}^{1} \mu(\xi)\left(\begin{array}{c}
K \\
k
\end{array}\right) p(\xi)^{k}(1-p(\xi))^{K-k} \quad \text { where } \quad p(\xi)=R^{*}\left(p_{0}+(1-\xi) p_{1}\right)
$$

where $\xi \in\{0,1\}$ and $\mu(1)=q, \mu(0)=1-q$. Figure 10 displays the recovery distribution with calibrated parameters $q$ as in [4], for the two different data sets CDX.NA.IG series sampled at 200712-07 and iTraxx Europe sampled at 2008-03-31. Here $\mathbb{E}[R]=R^{*}=0.4$ and $p_{0}=0.4$ in both cases. The calibrated parameter was $q=0.4405$ for the 2007 data set and $q=0.6002$ for the 2008 case.

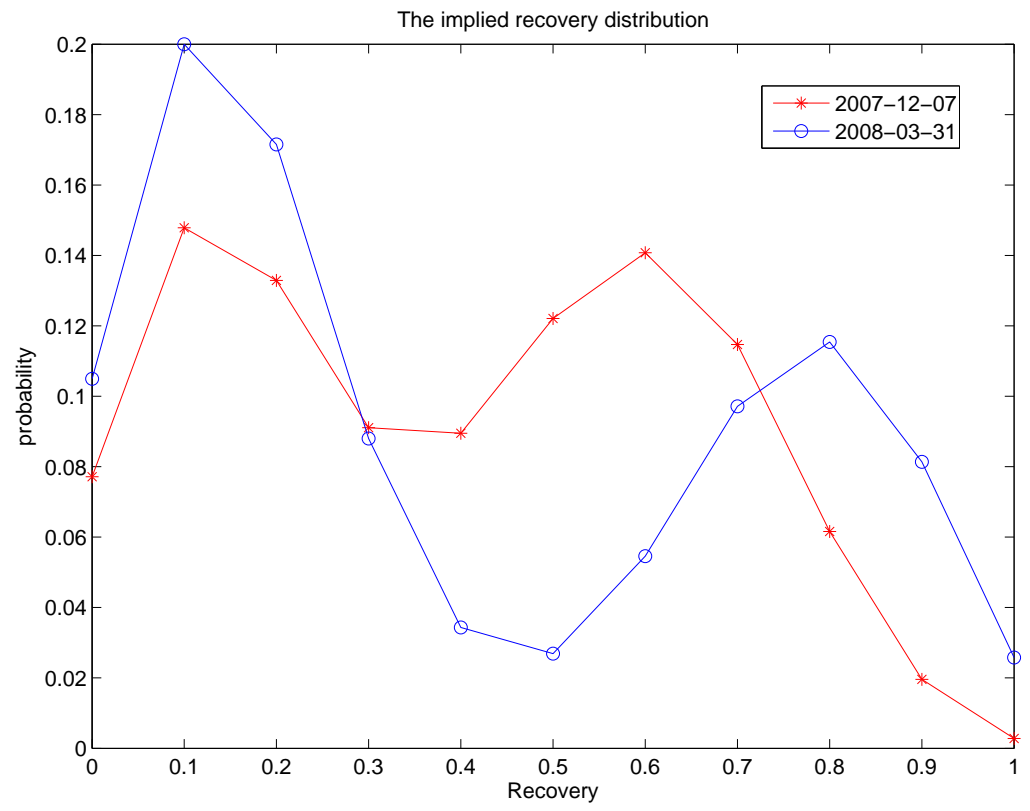

Figure 10: The implied recovery distribution with calibrated parameters $q$ in [4, for the two different data sets CDX.NA.IG series sampled at 2007-12-07 and iTraxx Europe sampled at 2008-03-31, where $\mathbb{E}[R]=R^{*}=0.4$ in both cases.

As seen in Figure 10, the implied probability for a recovery of $0 \%, 10 \%$ and $20 \%$ was consistenly higher in the 2008 sample compared with the 2007 data set (in March 2008 Bear Stearns was bailed out leading to around three times higher credit spreads than in December 2007, both in Europe and North America). Recall that a recovery of $0 \%$ means that everything is lost at a default.

In view of (49) and (50) we can give an explicit expression for the quantity $\mathbb{E} e^{\gamma\left(1-R_{i}\right)}$ specified in Subsection 3.3, as follows

$$
\mathbb{E} e^{\gamma\left(1-R_{i}\right)}=\sum_{\xi=0}^{1} \sum_{k=0}^{K} e^{\gamma\left(1-\frac{k}{K}\right)}\left(\begin{array}{l}
K \\
k
\end{array}\right) p(\xi)^{k}(1-p(\xi))^{K-k} .
$$

Recall that $\mathbb{E} e^{\gamma\left(1-R_{i}\right)}$ together with the corresponding computations in Subsection 3.3 and Equation (39) will determine the quantity $\mathbb{E} e^{\gamma_{\ell} \frac{L_{t}}{2 c}}$ in (37) which in turn is needed to compute the expected tranche loss given by Equation (36).

Let $R^{*}$ be a constant representing the average recovery for each obligor in the portfolio. We now impose the constraint $\mathbb{E}[R]=R^{*}$ which is necessary in order to have a calibration of the 
single-name CDSs that is separate from the calibration of the common-shock parameters. The condition $\mathbb{E}[R]=R^{*}$ leads to constraints on the parameters $p_{0}, q$ and $p_{1}$ that must be added to the constraints for the common shock intensities used in the calibration of the CDO tranches (recall that the calibration is a constrained minimization problem for these parameters). Below we derive these constraints for $p_{0}, q$ and $p_{1}$. First, note that

$$
\mathbb{E}[R]=\frac{R^{*}}{K} K \mathbb{E}\left[p_{0}+(1-\xi) p_{1}\right]=R^{*} p_{0}+(1-q) p_{1}
$$

so the condition $\mathbb{E}[R]=R^{*}$ implies $p_{0}+(1-q) p_{1}=1$ which yields

$$
p_{1}=\frac{1-p_{0}}{1-q} .
$$

Thus, $p_{1}$ can be seen as a function of $q$ and $p_{0}$. Next, in view of (49) we have for any scalar $\xi \in\{0,1\}$ that

$$
\mathbb{P}\left[R=\frac{k}{K} \mid \Theta=\xi\right]=\left(\begin{array}{c}
K \\
k
\end{array}\right) p(\xi)^{k}(1-p(\xi))^{K-k}
$$

where $p(\xi)$ is defined as in (50). Since $p(\xi)$ is a probability for $\xi \in\{0,1\}$ it must hold that

$$
p(1)=R^{*} p_{0} \in(0,1) \quad \text { and } \quad p(0)=R^{*}\left(p_{0}+p_{1}\right) \in(0,1)
$$

that is,

$$
0<R^{*} p_{0}<1 \text { and } 0<R^{*}\left(p_{0}+p_{1}\right)<1 .
$$

We can always assume that $p_{0}>0$ and $0<R^{*}<1$ so the first condition in (54) then implies

$$
p_{0}<\frac{1}{R^{*}} .
$$

Furthermore, by inserting (52) into the second condition in (54) we retrieve the following constraint

$$
0<R^{*} \frac{1-p_{0} q}{1-q}<1
$$

Since $q \in(0,1)$ and consequently $1-q>0$, then (56) implies $1-p_{0} q>0$, that is

$$
q<\frac{1}{p_{0}}
$$

However, we note that this is a "soft" condition since (55) implies that $p_{0}<\frac{1}{R^{*}}$ and if $p_{0}<1$ then (57) is superfluous since we already know that $0<q<1$. Next, (56) also tells us that $R^{*}\left(1-p_{0} q\right)<(1-q)$ which after some computation yields

$$
q<\frac{1-R^{*}}{1-R^{*} p_{0}} .
$$

Finally, it must obviously hold that $q<1$ since $\mathbb{P}[\Theta=1]=q$. Thus, combining this with (57) and (58) gives us the following final constraint for the parameter $q$,

$$
q<\min \left(1, \frac{1}{p_{0}}, \frac{1-R^{*}}{1-R^{*} p_{0}}\right) .
$$

This constraint was stated in 4 but without a proof. Furthermore, in [4 the constraint (59) was used in the calibration of the CDO tranches simultaneously with the other constraints for the common shock intensities. In [4] the parameters $p_{0}$ and $R^{*}$ are treated as exogenously given parameters with $R^{*}=40 \%$ while $p_{0}$ can be any positive scalar satisfying (55). The scalar $p_{0}$ will give some freedom to fine-tune the CDO calibrations, although it is of course possible to also let $p_{0}$ be another parameter used in the calibration. 


\section{Conclusions}

In this paper we make a focus on two practically important features of the Markov copula portfolio credit risk model of [4: random recoveries and stochastic intensities. Regarding random recoveries it would be interesting to find ways to add some dependence features without breaking the model tractability (in the current specifications one is only able to work with independent recoveries). As for stochastic intensities it would nice to find a good way of fixing the parameters $a$ and $c$, maybe based on historical observation of the dynamics of CDS spreads, rather than quite arbitrarily in this paper, as these dynamic parameters have little impact on CDS and CDO spreads. Also note that other specification of the intensities could be used, in particular Lévy Hull-White intensities driven by subordinators (for the sake of non-negativity, cf. Example 3.6 in [14]). Finally it would be interesting to apply these alternative specifications and to compare them in the context of CVA computations on portfolios of CDS and/or CDOs.

\section{References}

[1] Andersen, L. And Sidenius, J.: Extensions to the Gaussian Copula: Random Recovery and Random Factor Loadings, Journal of Credit Risk, Vol. 1, No. 1 (Winter 2004), p. 29-70.

[2] Assefa, S., Bielecki, T.R., Crépey, S. and Jeanblanc, M.: CVA computation for counterparty risk assessment in credit portfolios. Credit Risk Frontiers, Bielecki, T.R., Brigo, D. and Patras, F., eds., Wiley/Bloomberg-Press, 2011.

[3] Beylkin, G. And Monzon, L. On approximation of functions by exponential sums, Applied and Computational Harmonic Analysis, 19(1): 17-48, 2005.

[4] Bielecki, T.R., Cousin, A., Crépey, S., Herbertsson, A.: Dynamic Hedging of Portfolio Credit Risk in a Markov Copula Model, Submitted.

[5] Bielecki, T. R. And Crépey, S.: Dynamic Hedging of Counterparty Exposure. Forthcoming in The Musiela Festschrift, Zariphopoulou, T., Rutkowski, M. and Kabanov, Y., eds, Springer, 2012.

[6] Bielecki, T.R., Crépey, S. and Jeanblanc, M.: Up and Down Credit Risk.Quantitative Finance, 1469-7696, April 2010.

[7] Bielecki, T.R., Crépey, S., Jeanblanc, M. and Zargari, B.: Valuation and Hedging of CDS Counterparty Exposure in a Markov Copula Model. International Journal of Theoretical and Applied Finance, 15(1) 1250004 (39 pages), 2012.

[8] Bielecki, T. R. And Jakubowski, J. and Niewȩglowski, M.: Dynamic Modeling of Dependence in Finance via Copulae Between Stochastic Processes, Copula Theory and Its Applications, Lecture Notes in Statistics, Vol.198, Part 1, 33-76, 2010.

[9] Bielecki, T.R., Vidozzi, A. And Vidozzi, L.: A Markov Copulae Approach to Pricing and Hedging of Credit Index Derivatives and Ratings Triggered Step-Up Bonds, J. of Credit Risk, March 2008.

[10] Brigo D., Morini M. and Pallavicini, A.: Counterparty Credit Risk, Collateral and Funding with pricing cases for all asset classes. Forthcoming, Wiley, 2012.

[11] Brigo, D., Pallavicini, A., Torresetti, R. Credit models and the crisis: default cluster dynamics and the generalized Poisson loss model, Journal of Credit Risk, 6(4), 39-81, 2010.

[12] Brigo, D., Pallavicini, A., Torresetti, R.: Calibration of CDO Tranches with the dynamical Generalized-Poisson Loss model. Risk Magazine, May 2007.

[13] Brigo, D., Pallavicini, A., Torresetti, R. Cluster-based extension of the generalized poisson loss dynamics and consistency with single names. International Journal of Theoretical and Applied Finance, Vol 10, n. 4, 607-632, 2007. 
[14] Crépey, S., Grbac, Z. And Nguyen, H. N.: A multiple-curve HJM model of interbank risk. Mathematics and Financial Economics, Vol 6, Issue 6, p. 155-190, 2012.

[15] Crépey, S. and Rahal, A.: Simulation/Regression Pricing Schemes for CVA Computations on CDO Tranches. Submitted, 2012.

[16] Duffie, D. And GÂRleanu, N.: Risk and the valuation of collateralized debt obligations, Financial Analysts Journal, 57, 41-62, 2001.

[17] Elouerkhaoui, Y.: Pricing and Hedging in a Dynamic Credit Model. International Journal of Theoretical and Applied Finance, Vol. 10, Issue 4, 703-731, 2007.

[18] Herbertsson, A. (2008). Pricing synthetic CDO tranches in a model with Default Contagion using the Matrix-Analytic approach, The Journal of Credit Risk 4(4), 3-35.

[19] Iscoe, I., Jackson, K., Kreinin, A. And Ma, X.: An Exponential Approximation to the Hockey Stick Function. Working Paper, Department of Computer Science, University of Toronto, 2010.

[20] Iscoe, I., Jackson, K., Kreinin, A. And Ma, X.: Pricing Synthetic CDOs based on Exponential Approximations to the Payoff Function. Forthcoming in Journal of Computational Finance. 